\title{
Towards Sustainable and Reliable Networks with LIFETEL
}

\author{
Luca Chiaraviglio $^{1}$, Josip Lorincz ${ }^{2}$, Paolo Monti ${ }^{3}$ \\ ${ }^{1}$ DIET Department, Sapienza University of Rome, Rome, Italy, email luca.chiaraviglio@uniroma1.it \\ ${ }^{2}$ FESB-Split, University of Split, Split, Croatia, email josip.lerinc@ @esb.hr \\ ${ }^{3}$ Optical Networks Lab, KTH Royal Institute of Technology, Kista, Sweden, email pmonti@kth.se
}

\begin{abstract}
We present the LIFETEL project, whose aim is trying to increase the device lifetime in telecommunication networks by exploiting energy-efficient techniques. In particular, we show that the lifetime of a device might increase when it is put in sleep mode. However, the device lifetime might decrease when power state variations (from sleep mode to full power) occur very frequently. Thus, there is a trade-off between the duration of sleep mode and its frequency. Moreover, we report a lifetime analysis for cellular and backbone devices, showing that the electricity saving may be exceeded by the costs due to the fact that devices fail more frequently compared to the situation in which they are always powered on. Thus, we claim that energy-aware network algorithms should be redesigned in order not to impact and to increase (when possible) the lifetime of network devices.
\end{abstract}

\section{INTRODUCTION}

One of the most promising approaches to save energy in telecommunication networks is the adoption of sleep mode (SM). The idea of such technique is to put in a low-power state network devices (NDs) during off-peak hours. This intuition is corroborated by the fact that NDs hardly scale their consumed power with the load [1], therefore it becomes beneficial in terms of energy to completely switch them off.

Previous works have shown that SM is a promising approach to achieve energy-efficiency both in backbone [1], [2], [3], [4] and in cellular networks [5], [6]. However, current NDs do not fully support SM capabilities: this is due to the fact that operators are reluctant in applying this option. Their argument is quite strong: if NDs are not designed to be powered off, frequent power on/off may deteriorate the ND [7], leading to a probable failure, which is in general a traumatic event for a telecommunication network. In particular, SMs are related to failures since they affect the temperature of the ND. More in depth, when a SM state is set, the temperature on the ND is decreased, since few hardware (HW) components need to be powered on, while the largest part of its circuits is powered off. Intuitively, the lower is the temperature the lower will be also the probability of a failure event [8]. However, a variation of temperature is introduced in the device when passing from SM to full power and vice-versa. This temperature variation is particularly critical for HW components since it normally increases the failure rate (which is the inverse of the lifetime) [9]. Thus, we can see that there are two opposite effects triggered by the application of SM: one positive (decrease of temperature) and one negative (variation of temperature). LIFETEL (increasing the LIFEtime in TELecommunication networks) investigates this trade-off, by targeting the following questions: How much critical is the lifetime of NDs for an operator in terms of monetary costs? Which are the critical components for the lifetime of NDs? And also, is it possible to increase the lifetime of NDs when SM is used? The results of LIFETEL will pave the way towards sustainable and reliable telecommunication networks, where the application of SMs brings high energy savings together with fewer replacement of network equipment compared to the current situation.

\section{THE ENERGY-LIFETIME TRADE-OFF}

We define more formally the energy-lifetime trade-off investigated by LIFETEL. We assume that: i) the failure rate is intrinsically connected with the temperature of the device, ii) the change of power state (from SM to full power and viceversa) impacts the failure rate. Both these effects have been studied and modeled for memories and CPUs. In particular, a simple model that predicts the impact of the temperature on the failure rate is the Arrhenius law [10]: $\gamma^{\mathcal{T}}=\gamma^{0} e^{-\frac{E a}{K \mathcal{T}}}$, where $\gamma^{0}$ is the failure rate assuming infinite temperature, ${ }^{1} E_{a}$ is the activation energy (i.e., the minimum energy to trigger the process), $K$ is the Boltzmann constant, $\mathcal{T}$ is the measured temperature, and $\gamma^{\mathcal{T}}$ is the resulting failure rate. According to the Arrhenius law, we can see that that the lower is the temperature, the lower is the failure rate. However, the failure rate is also negatively affected by the frequency at which power state conditions vary. This effect is known as thermal cycling, and it is modeled by the Coffin-Manson equation [11]: $N^{f}=C_{0}\left(\Delta_{\mathcal{T}}-\Delta_{\mathcal{T}_{0}}\right)^{-q}$, where $\Delta_{\mathcal{T}}$ is the temperature variation, $\Delta_{\mathcal{T}_{0}}$ is variation of temperature supported by the device without an impact in the failure rate, $C_{0}$ is a material dependent constant, $q$ is the Coffin-Manson exponent, and $N^{f}$ is the number of cycles to failure. The failure rate due to thermal cycling is then defined as $\gamma^{\Delta_{\mathcal{T}}}=\frac{f^{T C}}{N^{f}}$ where $f^{T C}$ is the thermal cycling frequency and $\gamma_{\Delta_{\mathcal{T}}}$ is the resulting failure rate. Thus, an increase in the cycling frequency brings an increase in the failure rate. In our scenario, very frequent transitions between full power and SM will result in an increase in the failure rate.

\section{Lifetime Analysis: a CASE-Study}

To give a simple intution of the goal of the project, we provide some preliminary results obtained from backbone and cellular operator networks. In particular, Fig.1 reports the number of Base Station (BS) failures collected from a cellular network on 26th June 2014. We can clearly see that there are

\footnotetext{
${ }^{1}$ This term can be extracted by measuring the failure rate at very high temperatures.
} 


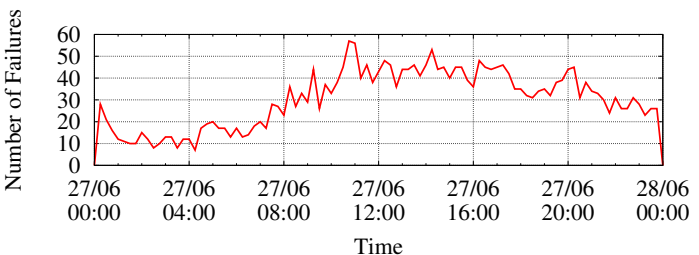

Fig. 1. Failures collected from a national cellular operator composed of more than 800 BSs.

TABle I. MaXimum Allowable Lifetime Decrease Analysis

\begin{tabular}{|l|l|l|l|l|l|l|}
\hline Device type & $\begin{array}{l}F R \\
{[\mathrm{FIT}]}\end{array}$ & $\begin{array}{l}M T T R \\
{[\mathrm{~h}]}\end{array}$ & Pers. & $\begin{array}{l}C_{e q} \\
{[\mathrm{kUSD}]}\end{array}$ & $\begin{array}{c}P_{e q} \\
{[\mathrm{~W}]}\end{array}$ & $L_{M A X_{10 \%}}$ \\
\hline $\begin{array}{l}\text { 100-Gbps Service } \\
\text { Line Card }\end{array}$ & 8600 & 2 & 1 & 190 & 130 & $0.18 \%$ \\
\hline $\begin{array}{l}\text { Macro BS (3 } \\
\text { sector UMTS, 2 } \\
\text { transceivers/sectror) }\end{array}$ & 10000 & 5 & 2 & 32.5 & 1700 & $7.33 \%$ \\
\hline
\end{tabular}

more failures experienced during the day w.r.t. the night, due to the high utilization of the network by the users. These failures result in an increase of the Operating Expenditures (OPEX) for the operator.

On the other hand, energy-efficient techniques exploiting the application of SMs may reduce the OPEX. In particular, the problem can be solved by applying algorithms which compute the set of devices to be powered off looking to the network as a whole (or at least to a portion of it) and by selecting a subset of devices to be powered on, thus bringing a saving in the electricity bill. However, if the device lifetime is not taken into account, the electricity saving may be exceeded by the costs due to the fact that devices fail more frequently compared to the situation in which they are always powered on.

To give more insight, we have estimated the monetary costs of failure rates and the monetary gains of SM for a linecard and macro BS. In particular, we have focused on the worst case scenario: a failure requiring the replacement of the device. In this scenario, we have estimated the maximum allowable lifetime decrease $\left(L D_{M A X}\right)$ introduced by SM. $L D_{M A X}$ is computed by imposing the difference of the failure costs (before and after SM is applied) to be equal to the monetary gain in terms of energy savings. Following the same reasoning, $L D_{M A X_{10 \%}}$ can be defined as the maximum allowable lifetime decrease so that the reparation costs will not exceed the saving obtained by lowering the energy consumption by $10 \%$. More formally, $L D_{M A X_{10 \%}}$ can be expressed in the following way:

$$
L D_{M A X_{10}}=\frac{\overbrace{10 \% \cdot P_{e q} \cdot C_{k W h}}^{\text {monetary energy saving }}}{\underbrace{10 \% \cdot P_{e q} \cdot C_{k W h}}_{\text {monetary energy saving }}+\underbrace{\frac{F R}{10^{6}} \cdot\left(M T T R \cdot P e r s . C_{m}+C_{e q}\right)}_{\text {reparation costs }}},
$$

where $P_{e q}[\mathrm{~W}]$ represents the power consumption at full power of the device, $C_{k W h}[\mathrm{USD} / \mathrm{kWh}]$ is the hourly electricity cost, $F R$ represents the device failure rate expressed in FIT [units] (i.e., the failure in time unit which corresponds to one failure per $10^{9}$ hours of operation), MTTR [h] is the mean time to repair the device, Pers. [member] represents the number of reparation crew members necessary to repair the failed device, $C_{m}[\mathrm{USD} / \mathrm{h} / \mathrm{member}]$ is the hourly rate cost of a reparation crew member, and $C_{e q}$ [USD] is the cost to buy a replacement unit of the device under reparation.

Tab. I reports the values obtained considering different devices, assuming $C_{k W h}=0.16 \mathrm{USD} / \mathrm{kWh}$ and $C_{m}=190 \mathrm{USD}$ per hour. The input parameters of Eq. (1) are obtained from discussion with operators. Interestingly, $L D_{M A X}$ ranges between $0.18 \%$ and $7.33 \%$ when energy-savings of $10 \%$ are allowed. This means that the lifetime of the device can be reduced at most to $0.18 \%-7.33 \%$ compared to the "always on" solution, otherwise monetary losses will be introduced. If we extend this value to the whole operator network, we can clearly see that SMs need to be carefully applied in the network in order to avoid monetary loss.

\section{CONClusions AND Future WORK}

We have presented the intuition behind LIFETEL project, whose aim it to increase the device lifetime by exploiting energy-efficient techniques. We have shown through two simple models that the duration of SMs and the frequency at which they occur impact the ND lifetime. Additionally, we have shown that failures are critical events that are affecting an operator network. Moreover, the energy-aware algorithms need to be carefully planned, otherwise monetary loss will be introduced due to the repair/replacement of the ND. LIFETEL will be devoted to the definition of new algorithms for increasing the device lifetime without impacting their reliability level. As future work, we will investigate such solutions in backbone and cellular networks.

\section{ACKNOWLEDGEMENTS}

The research leading to these results has received funding from the Sapienza Awards LIFETEL.

\section{REFERENCES}

[1] J. Chabarek, J. Sommers, P. Barford, C. Estan, D. Tsiang, and S. Wright, "Power awareness in network design and routing," in IEEE INFOCOM, Phoenix, USA, April 2008.

[2] L. Chiaraviglio, M. Mellia, and F. Neri, "Minimizing ISP network energy cost: Formulation and solutions," IEEE/ACM Transactions on Networking (TON), vol. 20, no. 2, pp. 463-476, 2012.

[3] R. Bolla, R. Bruschi, F. Davoli, and F. Cucchietti, "Energy efficiency in the future Internet: A survey of existing approaches and trends in energy-aware fixed network infrastructures," IEEE Communications Surveys \& Tutorials, vol. 13, no. 2, pp. 223-244, 2011.

[4] M. N. Dharmaweera, R. Parthiban, and Y. A. Şekercioğlu, "Towards a power-efficient backbone network: The state of research," IEEE Communications Surveys \& Tutorials, 2014.

[5] Z. Niu, Y. Wu, J. Gong, and Z. Yang, "Cell zooming for cost-efficient green cellular networks," IEEE Communications Magazine, vol. 48, no. 11, pp. 74-79, 2010.

[6] E. Oh, K. Son, and B. Krishnamachari, "Dynamic base station switching-on/off strategies for green cellular networks," IEEE Tran. on Wireless Communications, vol. 12, no. 5, pp. 2126-2136, 2013.

[7] P. Wiatr, J. Chen, P. Monti, and L. Wosinska, "Energy efficiency versus reliability performance in optical backbone networks," IEEE/OSA Journal of Optical Communication and Networking (JOCN), 2015.

[8] B. De Salvo, G. Ghibaudo, G. Pananakakis, G. Reimbold, F. Mondond, B. Guillaumot, and P. Candelier, "Experimental and theoretical investigation of nonvolatile memory data-retention," IEEE Transactions on Electron Devices, vol. 46, no. 7, pp. 1518-1524, 1999.

[9] A. K. Coskun, R. Strong, D. M. Tullsen, and T. Simunic Rosing, "Evaluating the impact of job scheduling and power management on processor lifetime for chip multiprocessors," in ACM SIGMETRICS/PERFORMANCE, Seattle, USA, pp. 169-180, 2009.

[10] S. Arrhenius, Über die Reaktionsgeschwindigkeit bei der Inversion von Rohrzucker durch Säuren. Wilhelm Engelmann, 1889.

[11] J. S. S. T. Association et al., "Failure mechanisms and models for semiconductor devices," JEDEC Publication JEP122-B, 2003. 